\title{
Design of New Amino Tf-Amide Organocatalysts: Environmental- ly Benign Approach to Asymmetric Aldol Synthesis
}

\author{
Hyo-Jun Lee ${ }^{a}$ \\ Natarajan Arumugamb \\ Abdulrahman I. Almansourb \\ Raju Suresh Kumar ${ }^{b}$ \\ Keiji Maruoka*a,c (i) \\ a Department of Chemistry, Graduate School of Science, Kyoto University, \\ Sakyo, Kyoto 606-8502, Japan \\ maruoka@kuchem.kyoto-u.ac.jp \\ ${ }^{b}$ Department of Chemistry, College of Science, King Saud University, \\ P.O. Box 2455, Riyadh, 11451, Saudi Arabia \\ ' School of Chemical Engineering and Light Industry, Guangdong University \\ of Technology, No.100, West Waihuan Road, HEMC, Panyu District, \\ Guangzhou, 510006, P. R. of China \\ Published as part of the 30 Years SYNLETT - Pearl Anniversary Issue
}

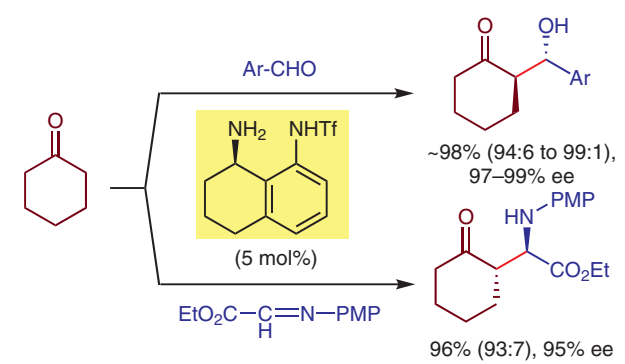

strategy is based on the enhancement of the reactivity of the acidic Tf-amide moiety by introducing an aromatic Tfamide moiety as illustrated in primary amino Tf-amide organocatalysts of type $\mathbf{5}$, which could accelerate asymmetric aldol and Mannich reactions.
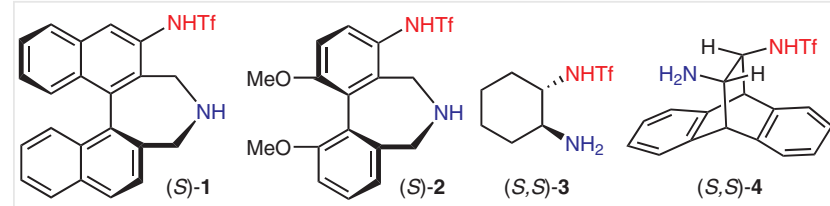

Key words asymmetric synthesis, aldol reaction, organocatalyst, brine, Mannich reaction

Among various types of amine-based, chiral, and bifunctional organocatalysts, amino Tf-amide organocatalysts are very reliable in various asymmetric transformations that include asymmetric aldol, Mannich, and conjugate addition reactions. ${ }^{1,2}$ In 2005, we reported the design of chiral binaphthyl-modified secondary amino Tf-amide organocatalysts of the type 1, which effectively promote anti-selective direct asymmetric Mannich reactions and syn-selective direct asymmetric cross-aldol reactions (Figure 1). ${ }^{3}$ Later, we prepared structurally similar chiral biphenyl-modified secondary amino Tf-amide organocatalysts of type $\mathbf{2 .}^{4}$ Moreover, we also designed primary amino Tf-amide organocatalysts of the type $\mathbf{3}$ and $\mathbf{4}$ for asymmetric aldol reactions and asymmetric conjugate additions. ${ }^{5,6}$ Compared to primary amino aliphatic Tf-amide organocatalysts of the type $\mathbf{3}$ and $\mathbf{4}$, which are not applicable to asymmetric Mannich-type reactions, the catalysts $\mathbf{1}$ and $\mathbf{2}$ exhibit a high nucleophilicity of the secondary amino moiety in addition to the acidic, aromatic Tf-amide hydrogen atom. However, due to the laborious synthesis of $\mathbf{1}$ and $\mathbf{2 , 3}$, the design and synthesis of easily accessible reactive amine Tf-amide organocatalysts represent a desirable research target., ${ }^{7,8}$ Our

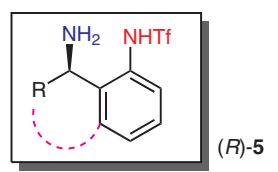

Figure 1 Previously and newly designed amino Tf-amide organocatalysts 1-5

Initially, we prepared various types of primary amino aromatic Tf-amide organocatalysts (6-10) and evaluated their reactivity and selectivity in asymmetric direct aldol reactions (Table 1 ). For this purpose, a series of aldol reactions between 4-nitrobenzaldehyde and cyclohexanone was carried out in the presence of catalysts $\mathbf{6 - 1 0}$ in aqueous THF at room temperature (Table 1, entries 1-5). ${ }^{9}$ Among these catalysts, 9 afford the highest stereoselectivity, giving the anti-aldol product (anti-11a) with $97 \%$ antiselectivity and $99 \%$ ee, albeit that $120 \mathrm{~h}$ are required for $60 \%$ yield (Table 1, entry 4 ). Subsequently, we carried out a solvent screening in order to improve the reactivity (Table 1 , entries 6-11). Among the solvents tested, aqueous solvents, in particular brine, dramatically accelerated the rate of the asymmetric aldol reactions (Table 1, entries 9 and 10), fur- 
nishing anti-11a in high yield with excellent stereoselectivity.$^{10}$ However, the low reactivity and selectivity were observed in the reaction in anhydrous DMSO (Table 1, entry 11 )..$^{5 b}$ Lowering the catalyst loading to $5 \mathrm{~mol} \%$ did not affect the anti- or enantioselectivity (Table 1, entry 12). However, carrying out the reaction with $2 \mathrm{~mol} \%$ of 9 slightly diminished the reactivity and afforded anti-11 with lower stereoselectivities (Table 1, entry 13).

Table 1 Optimization of the Catalysts and Reaction Conditions in the Asymmetric Direct Aldol Reactions between 4-Nitrobenzaldehyde and Cyclohexanone $^{\mathrm{a}}$
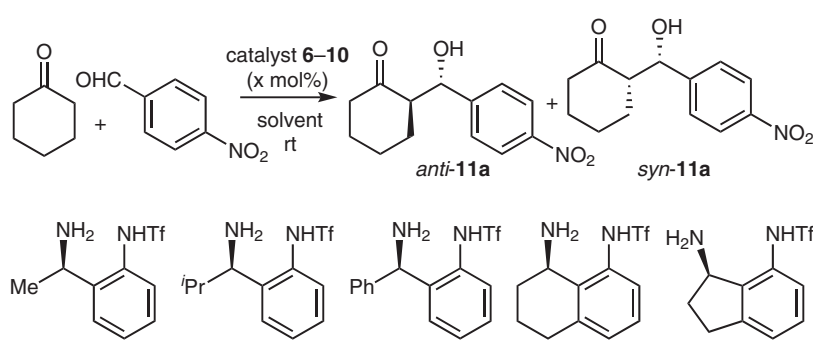

\begin{tabular}{|c|c|c|c|c|c|c|}
\hline Entry & $\begin{array}{l}\text { Catalyst } \\
\text { (x mol\%) }\end{array}$ & Solvent & $\begin{array}{l}\text { Time } \\
\text { (h) }\end{array}$ & $\begin{array}{l}\text { Yield } \\
(\%)^{\mathrm{b}}\end{array}$ & anti/syn ${ }^{c}$ & $\begin{array}{l}\text { ee } \\
(\%)^{d}\end{array}$ \\
\hline 1 & $6(10)$ & $\mathrm{THF} / \mathrm{H}_{2} \mathrm{O}$ & 168 & 32 & $75: 25$ & 89 \\
\hline 2 & $7(10)$ & $\mathrm{THF} / \mathrm{H}_{2} \mathrm{O}$ & 168 & 8 & $45: 55$ & 11 \\
\hline 3 & $8(10)$ & $\mathrm{THF} / \mathrm{H}_{2} \mathrm{O}$ & 48 & 96 & 90:10 & 97 \\
\hline 4 & $9(10)$ & $\mathrm{THF} / \mathrm{H}_{2} \mathrm{O}$ & 120 & 60 & $97: 3$ & 99 \\
\hline 5 & $10(10)$ & $\mathrm{THF} / \mathrm{H}_{2} \mathrm{O}$ & 120 & 96 & $93: 7$ & 86 \\
\hline 6 & $9(10)$ & $\mathrm{DMSO} / \mathrm{H}_{2} \mathrm{O}$ & 40 & 92 & $97: 3$ & 99 \\
\hline 7 & $9(10)$ & $\mathrm{EtOH} / \mathrm{H}_{2} \mathrm{O}$ & 120 & 72 & $87: 13$ & 83 \\
\hline 8 & $9(10)$ & neat & 24 & 69 & $94: 6$ & 99 \\
\hline 9 & $9(10)$ & $\mathrm{H}_{2} \mathrm{O}$ & 20 & 91 & $97: 3$ & 99 \\
\hline 10 & $9(10)$ & brine & 12 & 91 & $97: 3$ & 99 \\
\hline 11 & $9(10)$ & DMSO & 72 & 62 & $95: 5$ & 98 \\
\hline 12 & $9(5)$ & brine & 24 & 92 & $97: 3$ & 99 \\
\hline 13 & $9(2)$ & brine & 48 & 92 & $97: 3$ & 99 \\
\hline
\end{tabular}

a Unless otherwise specified, the asymmetric direct aldol reaction between cyclohexanone and 4-nitrobenzaldehyde was carried out in the presence of 2-10 mol\% of $\mathbf{6}-\mathbf{1 0}$ at room temperature.

${ }^{\mathrm{b}}$ Isolated yield.

' The anti/syn ratio was determined by ${ }^{1} \mathrm{H}$ NMR analysis.

$\mathrm{d} \%$ ee of major anti-isomer.

Subsequently, the scope of the asymmetric direct aldol reaction between cycloalkanones and various benzaldehydes in brine solvent was investigated under the optimized conditions using $5 \mathrm{~mol} \%$ of $\mathbf{9}$ (Table 2). ${ }^{11}$ The reactions involving benzaldehydes bearing strong electronwithdrawing substituent(s) at any position of the phenyl group furnished the corresponding aldol products $\mathbf{1 1}$ in high yields, with excellent anti- and enantioselectivity (Table 2, entries 1-7). Especially the heteroaromatic nicotinic and isonicotinic aldehydes afforded $\mathbf{1 1}$ in excellent yield and selectivity (Table 2, entries 8 and 9). Even though the reactivity for 4-halobenzaldehydes was slightly lower, these reactions still showed excellent stereoselectivity (Table 2 , entries 10 and 11). When benzaldehyde was employed, the corresponding aldol product $\mathbf{1 1 I}\left(\mathrm{Ar}=\mathrm{Ph} ; \mathrm{R}^{1}\right.$ and $\left.\mathrm{R}^{2}=-\left(\mathrm{CH}_{2}\right)_{4^{-}}\right)$ was obtained in moderate yield with high anti- and enantioselectivity (Table 2, entry 12). The use of cyclopentanone as the aldol donor also showed high reactivity and stereoselectivity for the corresponding aldol product $11 \mathrm{~m}(\mathrm{Ar}=4$ $\mathrm{NO}_{2}-\mathrm{C}_{6} \mathrm{H}_{4} ; \mathrm{R}^{1}$ and $\mathrm{R}^{2}=-\left(\mathrm{CH}_{2}\right)_{3}-;$ Table 2, entry 13). Cycloheptanone and acetone provided the corresponding aldol products in good yield with high stereoselectivity, though $10 \mathrm{~mol} \%$ of 9 were required (Table 2, entries 14 and 15).

Table 2 Asymmetric Direct Aldol Reaction of Cycloalkanone and Substituted Benzaldehydes Catalyzed by Organocatalyst $\mathbf{9}^{\text {a }}$

\begin{tabular}{|c|c|c|c|c|c|c|}
\hline Entry & $\operatorname{Ar}, R^{1}, R^{2}$ & 11 & $\begin{array}{l}\text { Time } \\
\text { (h) }\end{array}$ & $\begin{array}{l}\text { Yield } \\
(\%)^{\mathrm{b}}\end{array}$ & $\begin{array}{l}\text { an- } \\
\text { ti/sync }\end{array}$ & $\begin{array}{l}\text { ee } \\
(\%)^{d}\end{array}$ \\
\hline 1 & $4-\mathrm{NO}_{2}-\mathrm{C}_{6} \mathrm{H}_{4},-\left(\mathrm{CH}_{2}\right)_{4}-$ & $11 a$ & 24 & 92 & $97: 3$ & 99 \\
\hline 2 & $4-\mathrm{CF}_{3}-\mathrm{C}_{6} \mathrm{H}_{4},-\left(\mathrm{CH}_{2}\right)_{4}-$ & $11 b$ & 30 & 96 & $95: 5$ & 99 \\
\hline 3 & $4-\mathrm{CN}-\mathrm{C}_{6} \mathrm{H}_{4},-\left(\mathrm{CH}_{2}\right)_{4}-$ & $11 \mathrm{c}$ & 30 & 96 & $97: 3$ & 98 \\
\hline 4 & $4-\mathrm{CO}_{2} \mathrm{Me}-\mathrm{C}_{6} \mathrm{H}_{4},-\left(\mathrm{CH}_{2}\right)_{4}-$ & 11d & 48 & 88 & $97: 3$ & 99 \\
\hline 5 & $2-\mathrm{NO}_{2}-\mathrm{C}_{6} \mathrm{H}_{4},-\left(\mathrm{CH}_{2}\right)_{4}-$ & $11 e$ & 24 & 88 & $97: 3$ & 99 \\
\hline 6 & 3- $-\mathrm{NO}_{2}-\mathrm{C}_{6} \mathrm{H}_{4},-\left(\mathrm{CH}_{2}\right)_{4}-$ & $11 f$ & 48 & 84 & $98: 2$ & 99 \\
\hline 7 & $\mathrm{C}_{6} \mathrm{~F}_{5},-\left(\mathrm{CH}_{2}\right)_{4-}^{-}$ & $11 \mathrm{~g}$ & 30 & 88 & $99: 1$ & 97 \\
\hline 8 & 3-pyridyl, - $\left(\mathrm{CH}_{2}\right)_{4}-$ & $11 \mathrm{~h}$ & 36 & 92 & $96: 4$ & 99 \\
\hline 9 & 4-pyridyl, - $\left(\mathrm{CH}_{2}\right)_{4^{-}}$ & $11 i$ & 20 & 98 & $94: 6$ & 99 \\
\hline 10 & $4-\mathrm{Cl}-\mathrm{C}_{6} \mathrm{H}_{4},-\left(\mathrm{CH}_{2}\right)_{4}{ }^{-}$ & $11 \mathrm{j}$ & 48 & 55 & $97: 3$ & 99 \\
\hline 11 & $4-\mathrm{Br}-\mathrm{C}_{6} \mathrm{H}_{4},-\left(\mathrm{CH}_{2}\right)_{4}-$ & $11 \mathrm{k}$ & 48 & 72 & $97: 3$ & 99 \\
\hline 12 & $\mathrm{C}_{6} \mathrm{H}_{5},-\left(\mathrm{CH}_{2}\right)_{4^{-}}$ & 111 & 48 & 47 & $94: 6$ & 98 \\
\hline 13 & $4-\mathrm{NO}_{2}-\mathrm{C}_{6} \mathrm{H}_{4},-\left(\mathrm{CH}_{2}\right)_{3}-$ & $11 \mathrm{~m}$ & 18 & 89 & $91: 9$ & 99 \\
\hline $14^{\mathrm{e}}$ & $4-\mathrm{NO}_{2}-\mathrm{C}_{6} \mathrm{H}_{4},-\left(\mathrm{CH}_{2}\right)_{5}-$ & $11 n$ & 48 & 92 & $88: 12$ & 89 \\
\hline $15^{\mathrm{e}}$ & 4- $\mathrm{NO}_{2}-\mathrm{C}_{6} \mathrm{H}_{4}, \mathrm{CH}_{3}, \mathrm{H}$ & 110 & 48 & 80 & - & 87 \\
\hline
\end{tabular}

a Unless otherwise specified, the asymmetric direct aldol reaction between cycloalkanone and the substituted benzaldehydes was carried out in brine in the presence of $5 \mathrm{~mol} \%$ of 9 at room temperature.

${ }^{\mathrm{b}}$ Isolated yield.

' The anti/syn ratio was determined by ${ }^{1} \mathrm{H}$ NMR analysis.

$\mathrm{d} \%$ ee of major anti-isomer.

e $10 \mathrm{~mol} \%$ of 9 was used.

In order to demonstrate the applicability of the catalyst 9 in asymmetric transformations, we carried out asymmetric direct Mannich reaction between cyclic ketones and $\alpha$ imino ester $\mathbf{1 2}$ in the presence of catalytic amounts of $\mathbf{9}$ in 
THF at room temperature (Scheme 1), which afforded the anti-Mannich products 13a (anti/syn $=93: 7 ; 95 \%$ ee (anti)), 13b $($ anti $/$ syn $=96: 4 ; 97 \%$ ee $($ anti $)$, and 13c $($ anti/syn = 97:3; $92 \%$ ee $(a n t i))$ in high yield. ${ }^{12}$

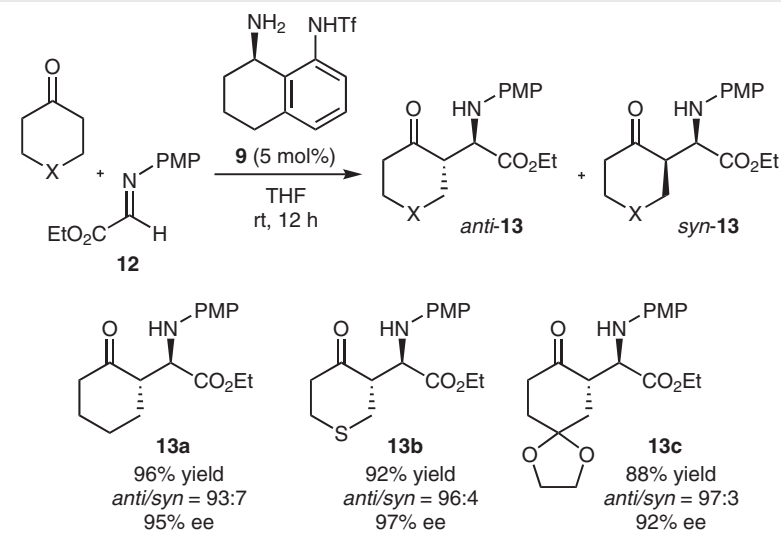

Scheme 1 Asymmetric Mannich reaction between cyclic ketone and $\alpha$-imino ester 13 catalyzed by organocatalyst 9 .

\section{Funding Information}

This work was partially supported by a Grant-in-Aid for Scientific Research from MEXT, Japan (Grant Number JP26220803, JP17H06450). The authors also extend their gratitude to the International Scientific Partnership Program (ISPP) at the King Saud University for financial support via ISPP\#0072.

\section{Supporting Information}

Supporting information for this article is available online at https://doi.org/10.1055/s-0037-1610408.

\section{References and Notes}

(1) Recent reviews on amine-based organocatalysts: (a) Kano, T.; Maruoka, K. Chem. Commun. 2008, 5465. (b) Lui, X.; Lin, L.; Feng, X. Chem. Commun. 2009, 6145. (c) Yang, H.; Carter, R. G. Synlett 2010, 2827. (d) Giacalone, F.; Gruttadauria, M.; Agrigento, P.; Noto, R. Chem. Soc. Rev. 2012, 41, 2406. (e) Melchiorre, P. Angew. Chem. Int. Ed. 2012, 52, 9748. (f) Kano, T.; Maruoka, K. Chem. Sci. 2013, 4, 907. (g) Duan, J.; Li, P. Catal. Sci. Technol. 2014, 4, 311. (h) Kumar, P.; Sharma, B. M. Synlett 2018, 29, 1994. (i) Zhu, L.; Wang, D.; Jia, Z.; Lin, Q.; Huang, M.; Luo, S. ACS Catal. 2018, 8, 5466.

(2) Recent reviews on amino-catalyzed asymmetric aldol and Mannich reactions: (a) Trost, B. M.; Brindle, C. S. Chem. Soc. Rev. 2010, 39, 1600. (b) Hernández, J. G.; Juaristi, E. Chem. Commun. 2012, 48, 5396. (c) Heravi, M. M.; Zadsirjan, V.; Dehghani, M.; Hosseintash, N. Tetrahedron: Asymmetry 2017, 28, 587. (d) Saranya, S.; Harry, N. A.; Krishnan, K. K.; Anilkumar, G. Asian J. Org. Chem. 2018, 7, 613. (e) Yamashita, Y.; Yasukawa, T.; Yoo, W.-J.; Kitanosono, T.; Kobayashi, S. Chem. Soc. Rev. 2018, 47, 4388.
(3) (a) Kano, T.; Yamaguchi, Y.; Tokuda, O.; Maruoka, K.J. Am. Chem. Soc. 2005, 127, 16408. (b) Kano, T.; Yamaguchi, Y.; Tanaka, Y.; Maruoka, K. Angew. Chem. Int. Ed. 2007, 46, 1738. (c) Kano, T.; Yamamoto, A.; Maruoka, K. Tetrahedron Lett. 2008, 49, 5369. (d) Kano, T.; Yamaguchi, Y.; Maruoka, K. Angew. Chem. Int. Ed. 2009, 48, 1838. (e) Kano, T.; Yamaguchi, Y.; Maruoka, K. Chem. Eur. J. 2009, 15, 6678. See also: (f) Kano, T.; Ueda, M.; Takai, J.; Maruoka, K. J. Am. Chem. Soc. 2006, 128, 6046.

(4) (a) Kano, T.; Sugimoto, H.; Maruoka, K. J. Am. Chem. Soc. 2011, 133, 18130. (b) Kano, T.; Song, S.; Maruoka, K. Angew. Chem. Int. Ed. 2012, 51, 1191. (c) Kano, T.; Song, S.; Maruoka, K. Chem. Commun. 2012, 48, 7037.

(5) (a) Nakayama, K.; Maruoka, K. J. Am. Chem. Soc. 2008, 130, 17666. (b) Moteki, S. A.; Maruyama, H.; Nakayama, K.; Li, H.-B.; Petrova, G.; Maeda, S.; Morokuma, K.; Maruoka, K. Chem. Asian. J. 2015, 10, 2112. (c) Lee, H.-J.; Moteki, S. A.; Arumugam, N.; Almansour, A. I.; Kumar, R. S.; Liu, Y.; Maruoka, K. Asian J. Org. Chem. 2017, 6, 1226. (d) Lee, H.-J.; Arumugam, N.; Almansour, A. I.; Kumar, R. S.; Maruoka, K. Tetrahedron 2018, 74, 5263.

(6) Moteki, S. A.; Xu, S.; Arimitsu, S.; Maruoka, K. J. Am. Chem. Soc. 2010, 132, 17074.

(7) The synthesis of the primary amino aromatic Tf-amide organocatalysts 6-10 was carried out according to Scheme 2 (see Supporting Information for details).

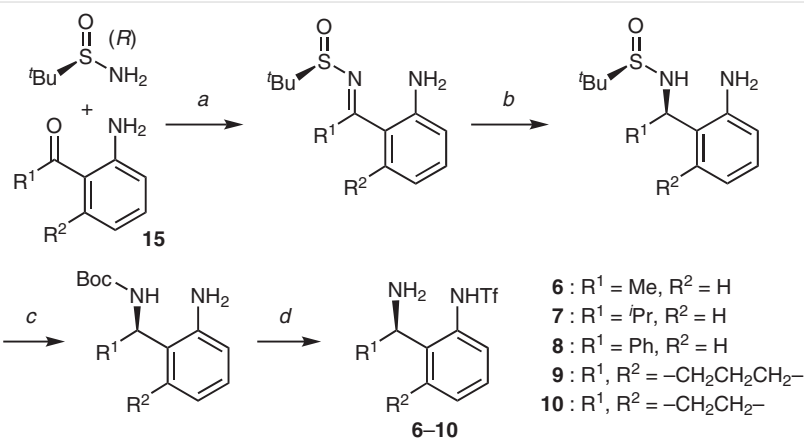

Scheme 2 Reagents and conditions: (a) Ti(OEt) 4 , THF, $70{ }^{\circ} \mathrm{C}$ or $90^{\circ} \mathrm{C}$; (b) DIBAL-H, THF, $-78^{\circ} \mathrm{C}$; (c) (i) $\mathrm{HCl}$, dioxane, $\mathrm{MeOH}$, rt; (ii) $\mathrm{Boc}_{2} \mathrm{O}, \mathrm{CH}_{2} \mathrm{Cl}_{2}$, rt; (d) (i) $\mathrm{Tf}_{2} \mathrm{O}, \mathrm{Et}_{3} \mathrm{~N}, \mathrm{CH}_{2} \mathrm{Cl}_{2},-78^{\circ} \mathrm{C}$; (ii) TFA, $\mathrm{CH}_{2} \mathrm{Cl}_{2}$, rt.

(8) The absolute configurations of the catalysts were assigned based on the X-ray diffraction analysis of the sulfonamide intermediate of catalyst $\mathbf{9 b}$. The corresponding data have been deposited at the Cambridge Crystallographic Data Center and can be obtained free of charge via www.ccdc.cam.ac.uk/getstructures. CCDC 1870339 contains the supplementary crystallographic data for this paper.

(9) Reviews of amino-catalyzed asymmetric aldol reaction in aqueous media: (a) Bhowmick, S.; Bhowmick, K. C. Tetrahedron: Asymmetry 2011, 22, 1945. (b) Kitanosono, T.; Kobayashi, S. Adv. Synth. Catal. 2013, 355, 3095. (c) Mlynarski, J.; Bas, S. Chem. Soc. Rev. 2014, 43, 557. (d) Bhowmick, S.; Mondal, A.; Ghosh, A.; Bhowmick, K. C. Tetrahedron: Asymmetry 2015, 26, 1215.

(10) Selected examples for asymmetric aldol reaction in water or brine: (a) Mase, N.; Nakai, Y.; Ohara, N.; Yoda, H.; Takabe, K.; Tanaka, J.; Barbas, C. F. III J. Am. Chem. Soc. 2006, 128, 734. (b) Ma, X.; Da, C.-S.; Yi, L.; Jia, Y.-N.; Guo, Q.-P.; Che, L.-P.; Wu, F.-C.; Wang, J.-R.; Li, W.-P. Tetrahedron: Asymmetry 2009, 20, 1419. (c) Miura, T.; Kasuga, H.; Imai, K.; Ina, M.; Tada, N.; Imai, N.; Itoh, A. Org. Biomol. Chem. 2012, 10, 2209. (d) Qiao, Y.; Chen, 
Q.; Lin, S.; Ni, B.; Headley, A. D. J. Org. Chem. 2013, 78, 2693. (e) Hu, X.-M.; Zhang, D.-X.; Zhang, S.-Y.; Wang, P.-A. RSC Adv. 2015, 5, 39557.

(11) General Procedure for the Asymmetric Aldol Reaction Using the Catalyst 9 for the Preparation of 11

To a mixture of catalyst $\mathbf{9}$ ( $3 \mathrm{mg}, 5 \mathrm{~mol} \%)$ and benzaldehyde $(0.2$ $\mathrm{mmol})$ in brine $(1.2 \mathrm{~mL})$ was added ketone $(6.0 \mathrm{mmol})$. The homogenous mixture was stirred at room temperature for the appropriate time until the reaction was completed (TLC). Then, a saturated $\mathrm{NH}_{4} \mathrm{Cl}$ solution was added, and the mixture was extracted with dichloromethane. The organic layer was dried over $\mathrm{Na}_{2} \mathrm{SO}_{4}$, filtered, and concentrated in vacuo. The residue was then purified by silica gel column chromatography $($ EtOAc/hexane $=1: 3)$ to afford the product 11 .

(R)-2-[(S)-Hydroxy(4-nitrophenyl)methyl]cyclohexan-1-one (anti-11a)

White solid. ${ }^{1} \mathrm{H} \mathrm{NMR}\left(\mathrm{CDCl}_{3}, 500 \mathrm{MHz}\right): \delta=8.22-8.11(\mathrm{~m}, 2 \mathrm{H})$, 7.52-7.49 (m, $2 \mathrm{H}), 4.90$ (d, $J=8.0 \mathrm{~Hz}, 1 \mathrm{H}), 4.07$, (br s, $1 \mathrm{H}$ ), 2.61-2.56 (m, $1 \mathrm{H}), 2.52-2.47(\mathrm{~m}, 1 \mathrm{H}), 2.40-2.33$, (m, $1 \mathrm{H})$, 2.14-2.08 (m, $1 \mathrm{H}), 1.85-1.80(\mathrm{~m}, 1 \mathrm{H}), 1.72-1.62(\mathrm{~m}, 1 \mathrm{H})$, 1.60-1.51 (m, $2 \mathrm{H}), 1.42-1.33(\mathrm{~m}, 1 \mathrm{H}) .{ }^{13} \mathrm{C}$ NMR $\left(\mathrm{CDCl}_{3}, 125\right.$ $\mathrm{MHz}): \delta=214.7,148.3,147.5,127.8,123.5,74.0,57.1,42.6$,
30.7, 27.6, 24.6. HRMS (ESI): $\mathrm{m} / \mathrm{z}$ calcd for $\mathrm{C}_{13} \mathrm{H}_{15} \mathrm{O}_{4} \mathrm{NNa}$ : 272.0893 [M + Na $]^{+}$; found: 272.0895. $[\alpha]_{\mathrm{D}}{ }^{24}-11.2\left(\mathrm{CHCl}_{3}, c 0.9\right.$, 99\% ee).

(12) General Procedure for the Asymmetric Mannich Reaction Using Catalyst 9 for the Preparation of 13

To a mixture of catalyst $\mathbf{9}$ ( $3 \mathrm{mg}, 5 \mathrm{~mol} \%$ ) and $\alpha$-imino ester $\mathbf{1 2}$ $(42 \mathrm{mg}, 0.2 \mathrm{mmol})$ in THF $(1.2 \mathrm{~mL})$ was added ketone $(6.0$ $\mathrm{mmol}$ ). The mixture was stirred at room temperature for $12 \mathrm{~h}$. Then, a saturated $\mathrm{NH}_{4} \mathrm{Cl}$ solution was added, and the mixture was extracted with EtOAc. The organic layer was dried over $\mathrm{Na}_{2} \mathrm{SO}_{4}$, filtered, and concentrated in vacuo. The residue was then purified by silica gel column chromatography (EtOAc/hexane $=1: 4)$ to afford the product 13 .

Ethyl $(R)-2-[(4-M e t h o x y p h e n y l) a m i n o]-2-[(S)-2-0 x o c y c l o-$ hexyl]-acetate (anti-13a)

Colorless oil. ${ }^{1} \mathrm{H}$ NMR $\left(\mathrm{CDCl}_{3}, 500 \mathrm{MHz}\right): \delta=6.77-6.73(\mathrm{~m}, 2 \mathrm{H})$, 6.64-6.61 (m, $2 \mathrm{H}), 4.24$ (br s, $1 \mathrm{H}), 4.18-4.10(\mathrm{~m}, 2 \mathrm{H}), 3.98$ (d, $J=4.5 \mathrm{~Hz}, 1 \mathrm{H}), 3.73(\mathrm{~s}, 3 \mathrm{H}), 3.12-3.08(\mathrm{~m}, 1 \mathrm{H}), 2.45-2.40(\mathrm{~m}, 1$ H), 2.35-2.29 (m, $1 \mathrm{H}), 2.13-2.09(\mathrm{~m}, 1 \mathrm{H}), 2.07-2.02(\mathrm{~m}, 1 \mathrm{H})$, 1.96-1.87 (m, $2 \mathrm{H}), 1.78-1.62(\mathrm{~m}, 2 \mathrm{H}), 1.21$ (t, $J=7.5 \mathrm{~Hz}, 3 \mathrm{H})$. ${ }^{13} \mathrm{C}$ NMR $\left(\mathrm{CDCl}_{3}, 125 \mathrm{MHz}\right): \delta=210.9,173.0,152.7,142.1,115.6$, 114.7, 61.1, 59.0, 55.7, 53.5, 41.8, 30.5, 26.8, 24.5, 14.1. HRMS (ESI): $m / z$ calcd for $\mathrm{C}_{17} \mathrm{H}_{23} \mathrm{O}_{4} \mathrm{NNa}$ : 328.1519 [M $\left.+\mathrm{Na}\right]^{+}$; found: 328.1526. $[\alpha]_{\mathrm{D}}{ }^{24}-22.4\left(\mathrm{CHCl}_{3}, c 0.7,95 \%\right.$ ee $)$. 